\title{
Novel deep intronic mutation in PLA2G6 causing early-onset Parkinson's disease with brain iron accumulation through pseudo-exon activation
}

\author{
Chiara Cavestro $^{1}$ - Celeste Panteghini ${ }^{1}$. Chiara Reale ${ }^{1}$ - Alessia Nasca ${ }^{1} \cdot$ Silvia Fenu $^{2}$ - Ettore Salsano ${ }^{2}$. \\ Luisa Chiapparini ${ }^{3} \cdot$ Barbara Garavaglia $^{1} \cdot$ Davide Pareyson $^{2} \cdot$ Ivano Di Meo $^{1} \cdot$ Valeria Tiranti $^{1}$
}

Received: 22 April 2021 / Accepted: 4 August 2021 / Published online: 13 August 2021

(c) The Author(s) 2021

\begin{abstract}
PLA2G6 is the causative gene for a group of autosomal recessive neurodegenerative disorders known as PLA2G6-associated neurodegeneration (PLAN). We present a case with early-onset parkinsonism, ataxia, cognitive decline, cerebellar atrophy, and brain iron accumulation. Sequencing of PLA2G6 coding regions identified only a heterozygous nonsense variant, but mRNA analysis revealed the presence of an aberrant transcript isoform due to a novel deep intronic variant (c.2035-274G $>$ A) leading to activation of an intronic pseudo-exon. These results expand the genotypic spectrum of PLAN, showing the paramount importance of detecting possible pathogenic variants in deep intronic regions in undiagnosed patients.
\end{abstract}

Keywords PLA2G6 · PLAN · NBIA · Early-onset Parkinson's disease · Pseudo-exon activation · Deep intronic variant

\section{Introduction}

PLA2G6-associated neurodegeneration (PLAN) is a heterogeneous group of rare autosomal recessive neurodegenerative disorders caused by mutations in the PLA2G6 gene [1]. This gene encodes for iPLA2 $\beta$, a group VIA calciumindependent A2 phospholipase, involved in phospholipids metabolism essential for maintaining cell membrane integrity. Depending on age of onset and clinical features, PLAN can be classified in three subtypes, including infantile neuroaxonal dystrophy (INAD), atypical neuroaxonal dystrophy (ANAD), and PARK14 autosomal recessive early-onset

Ivano Di Meo and Valeria Tiranti contributed equally to this work.

Ivano Di Meo

ivano.dimeo@istituto-besta.it

Valeria Tiranti

valeria.tiranti@istituto-besta.it

1 Unit of Medical Genetics and Neurogenetics, Fondazione IRCCS Istituto Neurologico Carlo Besta, Milan, Italy

2 Unit of Rare Neurodegenerative and Neurometabolic Diseases, Fondazione IRCCS Istituto Neurologico Carlo Besta, Milan, Italy

3 Unit of Neuroradiology, Fondazione IRCCS Istituto Neurologico Carlo Besta, Milan, Italy
Parkinson's disease (EOPD). INAD and ANAD typically occurred in childhood, often associated with cerebellar cortical atrophy and iron deposition in the brain, a condition known as neurodegeneration with brain accumulation type II (NBIA2) [2]. Contrariwise, EOPD onset is in early adulthood, typically associated with dystonia, rapid cognitive decline, psychosis, dysarthria, and pyramidal tract signs [3]. However, it has been increasingly reported that PLAN can manifest with intermediate phenotypes partially matching those classically associated with this disorder, thus preventing the identification of a precise genotype-phenotype correlation [4-6].

In general, genetic disorders causative mutations have been prevalently identified in exons and in RNA donor or acceptor splice sites. However, despite next generation sequencing (NGS) has revolutionized genetic testing, a considerable proportion of patients with a clinical diagnosis for a recessive condition have only one heterozygous mutation, suggesting the presence of not detected deep intronic variations. Intronic point mutations can activate pseudo-exons, such as intronic sequences flanked by apparently good-toconsensus acceptor and donor-site signals that are never recognized by the splicing machinery [7].

Here, we report the first case of PLAN caused by a combination of nonsense and deep intronic variants in PLA2G6 gene. 

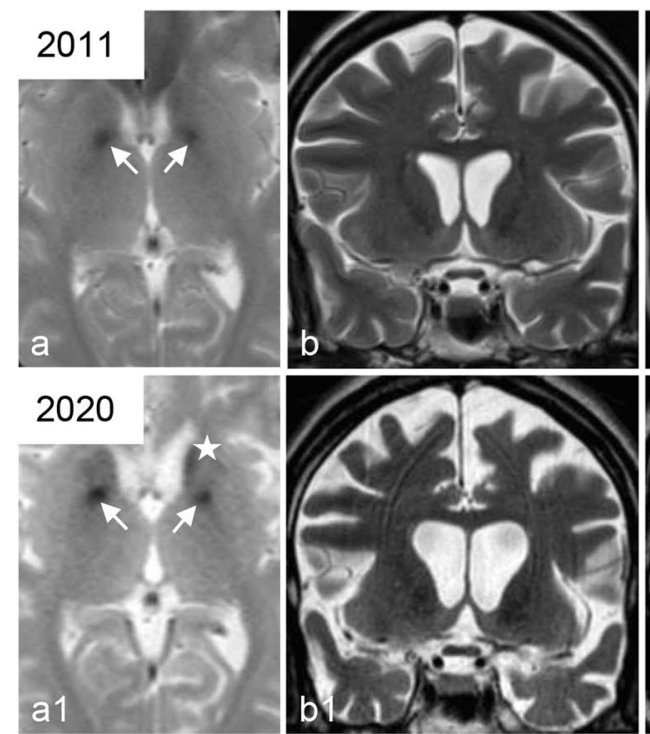
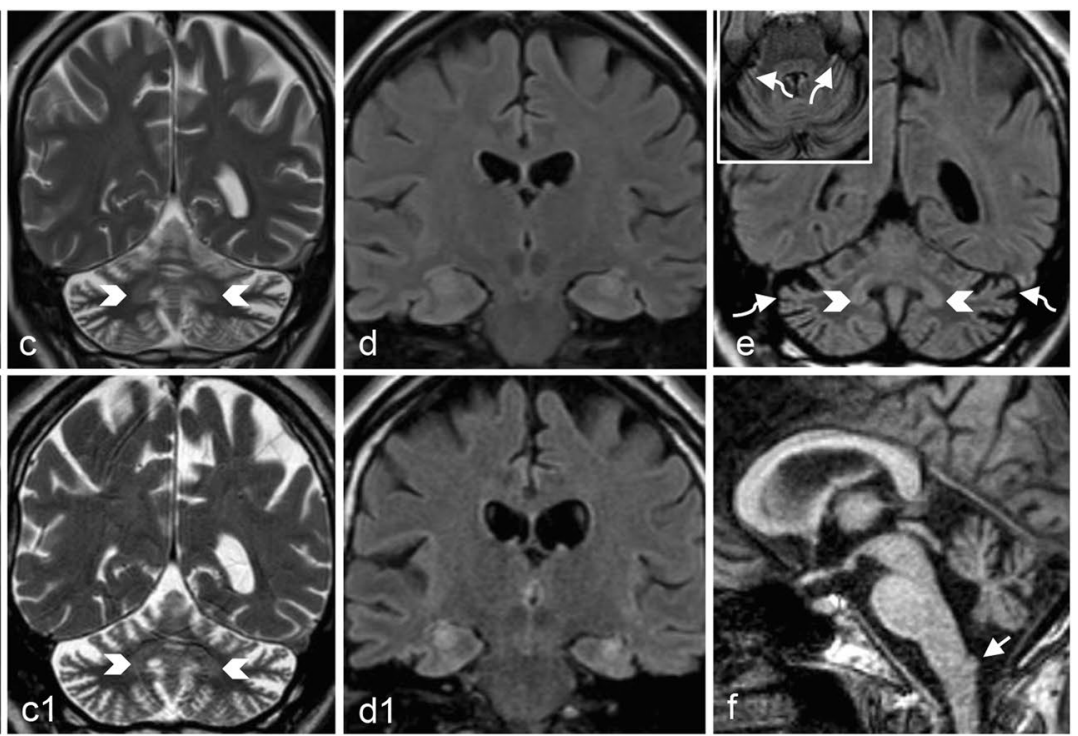

Fig. 1 Neuroradiological findings. Brain MRI performed at age 34 (a-d) and age 43 (a1-d1, e, and f) showing typical PLAN neuroradiological findings, except for not atrophic optic chiasm. a, a1 Axial T2 GRE images reveal, already in the first exam (a), globus pallidus hypointensity due to iron deposition (arrows); last examination (a1) demonstrates also iron in the head of the caudate nuclei (asterisk). b, b1, c, c1 Coronal T2-wi demonstrate normal optic chiasm volume and progressive diffuse cerebral atrophy (b and b1), associated with mild dentate nucleus hyperintensity (arrowheads in c, $\mathbf{c 1 ,}$, and e) and progressive cerebellar atrophy characterized by widening of cerebellar folia. d, d1 Coronal FLAIR images show an unusual atrophy and hyperintensity of both hippocampi in the last exam compared to the first. e Coronal and axial FLAIR image demonstrate cerebellar cortical hyperintensity (curved arrows). f Midline sagittal T1-wi shows cerebellar vermian atrophy and clava hypertrophy (arrow)

\section{Materials and methods}

This study was approved by the Ethics Committee of the Besta Institute with an informed, written consent. For sequencing of PLA2G6 transcript, PCR products were processed with Nextera XT DNA sample kit (Illumina) [8]. Real-time quantitative PCR (qPCR) was performed in a CFX-96 system (Bio-Rad) using iTaq Universal SYBR Green Supermix (Bio-Rad), with a primer pair specific for PLA2G6; the ACTB gene was used as reference. In silico predictors were used to assess the effect of variants on splicing (SpliceAI, HSF, NetGene2, NNSplice, varSEAK, MaxEntScan) [9]. The c.2035-274G $>$ A variant was submitted to the Leiden Open Variation Database (DB-ID: PLA2G6_000182).

\section{Results}

\section{Clinical reports}

The patient is a 43-year-old lady born to non-consanguineous parents and with no family history of neurological diseases. Her symptoms started at age 32 years with gait ataxia and scanned speech, and, since age 36 , a progressive asymmetric parkinsonian syndrome, with rigidity, hypokinesia, resting and postural tremor predominantly on the right, and cognitive decline. Response to L-Dopa was poor. She later developed behavioral abnormalities-irritability and occasional aggressiveness-treated with quetiapine. She progressively lost walking ability and developed dysphagia and severe akinesia.

At 43 years, examination revealed severe cognitive decline in memory, linguistic, frontal-executive, and visualspatial skills; pursuit saccadization, vertical upward gaze palsy, marked hypomimia, almost absence of spontaneous speech, which was scanned and monotonous, dysphagia, and drooling; severely hypokinetic gait, with double support; marked axial and limb rigidity, with trochlea sign, and generalized akinesia; oromandibular and bilateral upper limb resting tremor; increased deep tendon reflexes; and bilateral Babinski sign.

Brain MRI showed T2 GRE hypointensity of the pallida, substantia nigra and head of the left caudate nucleus, cerebellar atrophy, T2 hyperintensity of cerebellar cortex and dentate nuclei, and clava hypertrophy, and diffuse cerebral atrophy with hyperintensity of the hippocampi (Fig. 1).

EEG showed some slow abnormalities in the temporal regions, more pronounced on the left side, photosensitivity, 
a

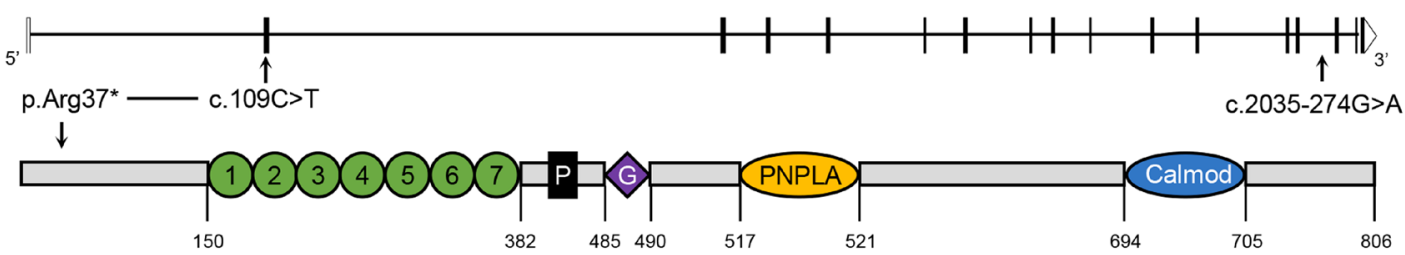

b

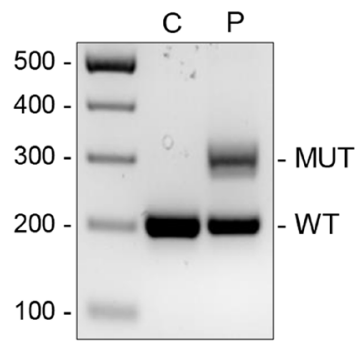

d

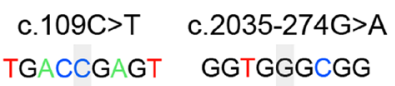

Patient
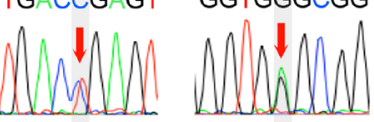

Mother
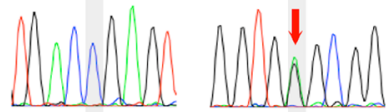

Father
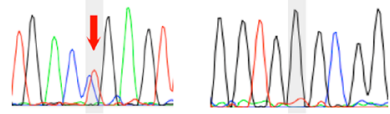

c

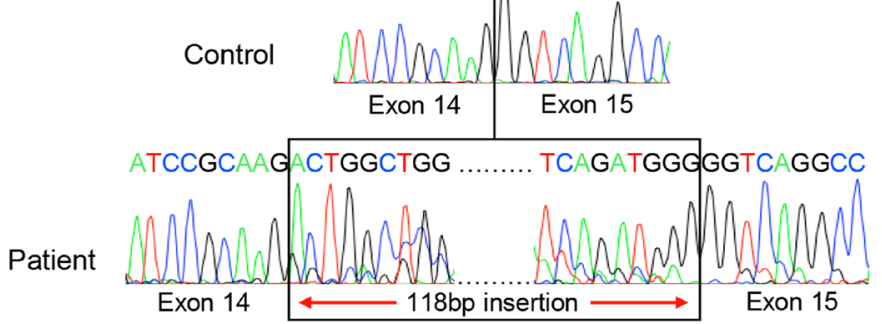

e

ATCCGCA

f

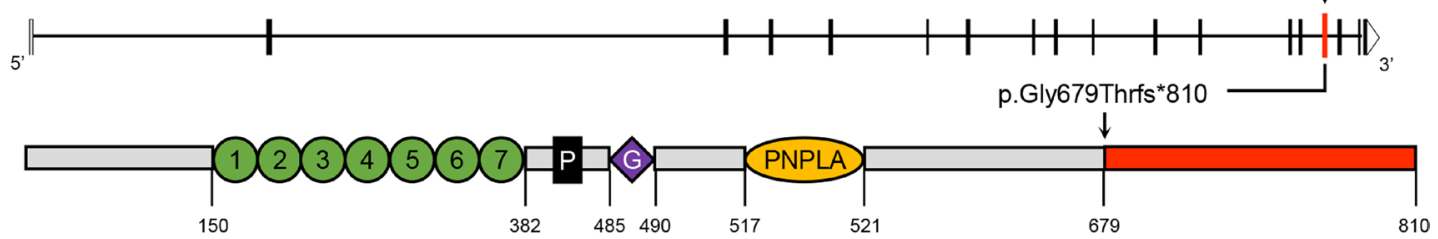

g

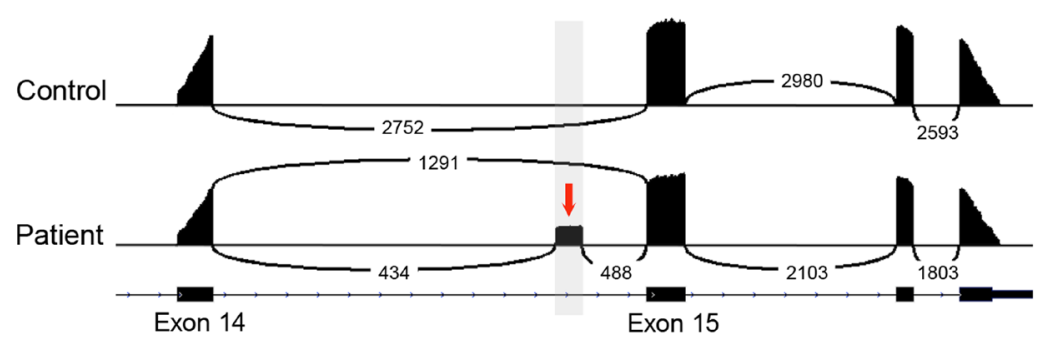

h

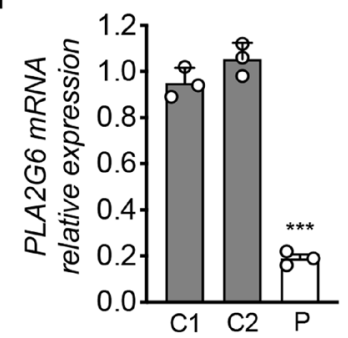

Fig. 2 Molecular studies. a Schematic representation of the PLA2G6 gene (upper) and protein (lower), and location of variants identified in this study. Protein consists of seven ankyrin repeats (numbered circles), a proline-rich motif (P), a glycine-rich nucleotide binding motif (G), a lipase motif (PNPLA), and a binding site for calmodulin (Calmod). Numbers shown below are the amino acid positions. b Amplicons spanning exons $14-15$ of the patient $(\mathrm{P})$ and a healthy control (C) show a different-sized PCR product in the patient sample. c Sanger sequencing of PLA2G6 cDNA shows inclusion of a 118-nucleotide intronic sequence between exons 14 and 15 (boxed) in patient. d Sanger sequencing of the PLA2G6 gene show compound heterozygous variants. The c. $109 \mathrm{C}>\mathrm{T}$ variant is inherited from the father, the c.2035-274G > A variant is from the mother. e Schematic of PLA2G6 exons 14-15 showing the c.2035-274G > A variant, which substitutes a less favored $\mathrm{G}$ (WT) at the +4 position for a highly favored A (MUT), strengthens a naturally occurring cryptic donor splice site to activate spliceosomal inclusion of the intron 14 pseudo-exon (boxed region). f Schematic representation of PLA2G6 gene and protein resulting from pseudo-exon inclusion. g Sashimi plots of PLA2G6 cDNA sequencing data show the presence of intron 14 pseudo-exon in patient, representing about $43 \%$ of total transcript. h Relative PLA2G6 mRNA expression in control $(\mathrm{C} 1, \mathrm{C} 2)$ and patient $(\mathrm{P})$ fibroblasts. Mean of three independent experiments $\pm \mathrm{SD}$ is shown. ${ }^{* * *} p<0.001$ (Student's $t$ test) 
Table 1 Splicing predictions of c.2035-274G > A variant by multiple bioinformatic prediction tools

\begin{tabular}{|c|c|c|c|c|}
\hline \multirow[t]{2}{*}{ Prediction tool } & \multicolumn{2}{|l|}{ Acceptor gain site } & \multicolumn{2}{|l|}{ Donor gain site } \\
\hline & Wild-type score & Mutant score & Wild-type score & Mutant score \\
\hline SpliceAI & n.a & 0.67 & n.a & 0.64 \\
\hline HSF & 85.21 & 85.21 & 79.82 & 90.1 \\
\hline NetGene2 & 0.22 & 0.22 & - & 0.64 \\
\hline NNSplice & 0.66 & 0.66 & - & 0.61 \\
\hline varSEAK & n.a & n.a & -48.06 & +29.04 \\
\hline MaxEntScan & 7.49 & 7.49 & 1.95 & 5.97 \\
\hline
\end{tabular}

n.a. not available and photomyoclonic response, without clinical evidence of seizures. There was latency prolongation of the central components of somatosensory evoked potentials.

\section{Molecular investigations}

Sequencing of PLA2G6 coding and flanking intron sequences revealed heterozygosity for the nonsense variant c.109C > T in exon 2 of the NM_003560.4 transcript (Fig. 2a), which introduces a premature stop codon at position 37 of the iPLA2 $\beta$ protein (p.Arg37*). This variant is reported as pathogenic in ClinVar database (accession: RCV000023318.6) and already associated with PLAN [10, 11]. However, we could find neither a second variant in the coding regions or adjacent introns nor the presence of exon deletion or duplication in PLA2G6 by MLPA analysis. Therefore, we isolated RNA from patient's fibroblasts and retrotranscribed it into cDNA to search for a second variant. Using intron-spanning primers pairs, we amplified an abnormal band of higher molecular weight in the patient that was not detected in controls (Fig. 2b). Sequencing of patient's PLA2G6 transcript revealed the inclusion of a $118 \mathrm{nt}$ region belonging to intron 14 (Fig. 2c). Then, we performed Sanger sequencing to explore the noncoding regions flanking the included sequence and identified a heterozygous deep intronic variant at position chr22. hg19:38,509,935 (c.2035-274G > A) (Fig. 2d, e), which was absent in the Genome Aggregation Database (gnomAD). In silico algorithms predicted the c.2035-274G $>$ A variant generates a pseudo-exon activation, gaining a novel splicing donor site $118 \mathrm{nt}$ downstream of a preexisting cryptic acceptor site (Fig. 2e, Table 1). The pseudo-exon inclusion causes a frameshift mutation (p.Gly679Thrfs*810), leading the complete loss of calmodulin domain (Fig. 2f). Sequencing of parents' genomic DNA confirmed that c.109C $>$ T and c. 2035-274G $>$ A variants were transmitted from father and mother, respectively (Fig. 2d). By NGS, we found that the isoform with the pseudo-exon inclusion represents about 43\% of patient's total PLA2G6 transcript (Fig. $2 \mathrm{~g}$ ). Likewise, the nonsense c. $109 \mathrm{C}>\mathrm{T}$ variant is present in about $55 \%$ of
mRNA (not shown). Moreover, qPCR performed on cDNA from patient and age-matched control fibroblasts showed a reduction of about $80 \%$ in the expression level of PLA2G6 transcript (Fig. 2h), suggesting that both variants lead to nonsense-mediated mRNA decay.

\section{Discussion}

It has been estimated that approximately half of the patients affected by rare genetic diseases remains without a definite molecular diagnosis, about $10 \%$ of which is due to pathogenic variants located deep within introns [7]. Here, we reported for the first time a deep intronic mutation in the PLA2G6 gene, causing the creation of a new donor splice site leading to a pseudo-exon inclusion by activating a preexisting cryptic acceptor splice site. This case, in which only one coding variant was detected at first, highlights that the existence of putative dominant variants in PLA2G6 should be reexamined [12]. Moreover, different studies reported that a consistent fraction of cases, ranging from 8 to $45 \%$, were heterozygous for a single PLA2G6 mutant allele, missing the second mutation [10,13-15]. Our patient's presentation is consistent with the rare phenotype observed in young adults, with atypical findings such as the T2 GRE hypointensity in the left caudate nucleus head and T2 hyperintensity in both hippocampi. Residual PLA2G6 activity may explain later symptom onset. To the best of our knowledge, the molecular mechanism here identified has never been described in genetic forms of Parkinson's disease. Furthermore, this case underlines the importance of cDNA analysis for detection of mutations in the intronic sequence of candidate genes in instances where exon sequencing and MLPA fail to provide a conclusive diagnosis. Although whole genome sequencing is entering faster and faster in the clinical diagnostic, RNA analysis offers a valid alternative for selected cases, although it may present limitations linked to sample availability, tissue-specific gene expression, or mutations inducing complete nonsensemediated mRNA decay. 
Acknowledgements We are grateful to the patient's family for participating in this work. We thank the "Cell line and DNA bank of movement disorders and mitochondrial diseases" of the Telethon Genetic Biobank Network (http://biobanknetwork.telethon.it). VT is a member of the European Reference Network for Rare Neuromuscular Diseases (ERN EURO-NMD). BG is a member of the European Reference Network for Rare Neurological Diseases (ERN- RND).

Funding This study was funded by Telethon-Italia (grant n. GGP16234 to V.T.) and by Italian Ministry of Health (grant n. GR-2018-12365610 to I.D.M.). This study was carried out in the Center for the Study of Mitochondrial Paediatric Diseases (http://www.mitopedia.org) sustained by the Mariani Foundation, Milan, Italy.

\section{Declarations}

Conflict of interest The authors declare no competing interests.

Open Access This article is licensed under a Creative Commons Attribution 4.0 International License, which permits use, sharing, adaptation, distribution and reproduction in any medium or format, as long as you give appropriate credit to the original author(s) and the source, provide a link to the Creative Commons licence, and indicate if changes were made. The images or other third party material in this article are included in the article's Creative Commons licence, unless indicated otherwise in a credit line to the material. If material is not included in the article's Creative Commons licence and your intended use is not permitted by statutory regulation or exceeds the permitted use, you will need to obtain permission directly from the copyright holder. To view a copy of this licence, visit http://creativecommons.org/licenses/by/4.0/.

\section{References}

1. Guo Y, Tang B, Guo J (2018) PLA2G6-associated neurodegeneration (PLAN): review of clinical phenotypes and genotypes. Front Neurol 9: https://doi.org/10.3389/fneur.2018.01100

2. Di Meo I, Tiranti V (2018) Classification and molecular pathogenesis of NBIA syndromes. Eur J Paediatr Neurol. https://doi. org/10.1016/j.ejpn.2018.01.008

3. Paisan-Ruiz C, Bhatia KP, Li A et al (2009) Characterization of PLA2G6 as a locus for dystonia-parkinsonism. Ann Neurol 65:19-23. https://doi.org/10.1002/ana.21415

4. Erro R, Balint B, Kurian MA et al (2016) Early ataxia and subsequent parkinsonism: PLA2G6 mutations cause a continuum rather than three discrete phenotypes. Mov Disord Clin Pract 4:125-128. https://doi.org/10.1002/mdc3.12319
5. Chu Y-T, Lin H-Y, Chen P-L, Lin C-H (2020) Genotype-phenotype correlations of adult-onset PLA2G6-associated neurodegeneration: case series and literature review. BMC Neurol 20: https://doi.org/10.1186/s12883-020-01684-6

6. Ji Y, Li Y, Shi C et al (2019) Identification of a novel mutation in PLA2G6 gene and phenotypic heterogeneity analysis of PLA2G6-related neurodegeneration. Parkinsonism Relat Disord 65:159-164. https://doi.org/10.1016/j.parkreldis.2019.04.002

7. Vaz-Drago R, Custódio N, Carmo-Fonseca M (2017) Deep intronic mutations and human disease. Hum Genet 136:10931111. https://doi.org/10.1007/s00439-017-1809-4

8. Legati A, Zanetti N, Nasca A et al (2021) Current and new nextgeneration sequencing approaches to study mitochondrial DNA. J Mol Diagn. https://doi.org/10.1016/j.jmoldx.2021.03.002

9. Jaganathan K, Kyriazopoulou Panagiotopoulou S, McRae JF et al (2019) Predicting splicing from primary sequence with deep learning. Cell 176:535-548.e24. https://doi.org/10.1016/j.cell. 2018.12.015

10. Morgan NV, Westaway SK, Morton JEV et al (2006) PLA2G6, encoding a phospholipase A 2 , is mutated in neurodegenerative disorders with high brain iron. Nat Genet 38:752-754. https://doi. org/10.1038/ng 1826

11. Tonelli A, Romaniello R, Grasso R et al (2010) Novel splice-site mutations and a large intragenic deletion in PLA2G6 associated with a severe and rapidly progressive form of infantile neuroaxonal dystrophy. Clin Genet 78:432-440. https://doi.org/10.1111/j. 1399-0004.2010.01417.x

12. Ferese R, Scala S, Biagioni F, et al (2018) Heterozygous PLA2G6 mutation leads to iron accumulation within basal ganglia and Parkinson's disease. Front Neurol 9: https://doi.org/10.3389/fneur. 2018.00536

13. Gregory A, Westaway SK, Holm IE et al (2008) Neurodegeneration associated with genetic defects in phospholipase A2. Neurology 71:1402-1409. https://doi.org/10.1212/01.wnl.0000327094. 67726.28

14. Zhang P, Gao Z, Jiang Y et al (2013) Follow-up study of 25 Chinese children with PLA2G6-associated neurodegeneration. Eur J Neurol 20:322-330. https://doi.org/10.1111/j.1468-1331.2012. 03856.x

15. Kapoor S, Shah MH, Singh N, et al (2016) Genetic analysis of PLA2G6 in 22 Indian families with infantile neuroaxonal dystrophy, atypical late-onset neuroaxonal dystrophy and dystonia parkinsonism complex. PLoS One 11: https://doi.org/10.1371/ journal.pone.0155605

Publisher's note Springer Nature remains neutral with regard to jurisdictional claims in published maps and institutional affiliations. 